\title{
Three-loop helicity amplitudes for diphoton production in gluon fusion
}

\author{
Piotr Bargieła, ${ }^{a}$ Fabrizio Caola, ${ }^{a, b}$ Andreas von Manteuffel ${ }^{c}$ and Lorenzo Tancredi ${ }^{a, d}$ \\ ${ }^{a}$ Rudolf Peierls Centre for Theoretical Physics, University of Oxford, \\ Clarendon Laboratory, Parks Road, Oxford OX1 3PU, U.K. \\ ${ }^{b}$ Wadham College, University of Oxford, \\ Parks Road, Oxford OX1 3PN, U.K. \\ ${ }^{c}$ Department of Physics and Astronomy, Michigan State University, \\ East Lansing, MI 48824, U.S.A. \\ ${ }^{d}$ Physik Department, Technische Universität München, \\ James-Franck-Straße 1, D-85748 Garching, Germany \\ E-mail: piotr.bargiela@physics.ox.ac.uk, \\ fabrizio.caola@physics.ox.ac.uk, vmante@msu.edu, \\ lorenzo.tancredi@tum.de
}

ABSTRACT: We present a calculation of the helicity amplitudes for the process $g g \rightarrow \gamma \gamma$ in three-loop massless QCD. We employ a recently proposed method to calculate scattering amplitudes in the 't Hooft-Veltman scheme that reduces the amount of spurious non-physical information needed at intermediate stages of the computation. Our analytic results for the three-loop helicity amplitudes are remarkably compact, and can be efficiently evaluated numerically. This calculation provides the last missing building block for the computation of NNLO QCD corrections to diphoton production in gluon fusion.

KEYwords: NLO Computations, QCD Phenomenology

ArXiv EPrint: 2111.13595 


\section{Contents}

1 Introduction 1

2 Notation and kinematics 2

3 The helicity amplitudes $\quad 4$

4 Details of the calculation $\quad 7$

5 UV renormalisation and IR regularisation 10

6 Checks and structure of the result 12

$\begin{array}{llr}7 & \text { Conclusions } & 14\end{array}$

\section{Introduction}

The production of two hard photons is an important process at hadron colliders, which both allows for scrutiny of the structure of the Standard Model and serves as an important background for many Higgs and new physics analyses.

From a theoretical perspective, the $p p \rightarrow \gamma \gamma$ process is rather peculiar. Phenomenologically, this process is interesting because an operative definition of isolated photons is non-trivial, and it requires quite subtle theoretical analysis [1]. Computationally, diphoton production is relatively simple, yet non-trivial. Indeed, photons are massless and colour-neutral particles, which implies that both the infrared structure and the scattering amplitudes for the diphoton process are not very complicated. However, compared to other colour-singlet processes like Higgs or Drell-Yan production, the kinematics of $\gamma \gamma$ production is more involved as it depends non-trivially on a scattering angle already at leading order $(\mathrm{LO})$ in the perturbative expansion. Because of these features, diphoton production is an ideal process for testing and improving our understanding of Quantum Chromodynamics (QCD) at hadron colliders. Indeed, historically $\gamma \gamma$ production has often served as a testing ground for innovative studies in perturbative QCD. For example, $\gamma \gamma$ production was the first hadron collider process with non-trivial LO kinematics for which next-to-next-to-leading order (NNLO) QCD corrections were computed [2]. Furthermore, $q \bar{q} \rightarrow \gamma \gamma$ was the first $2 \rightarrow 2$ QCD scattering amplitude that was calculated at the threeloop level [3]. Photon processes also played a prominent role in the development of NNLO predictions for $2 \rightarrow 3$ collider reactions [4-11].

The leading mechanism for producing two photons at hadron colliders is through $q \bar{q}$ annihilation. The availability of the two-loop QCD scattering amplitudes for $q \bar{q} \rightarrow \gamma \gamma$ [12] enabled detailed phenomenological predictions at NNLO accuracy [2, 13-17]. Starting 
from NNLO, the $g g$ partonic channel opens up. There are two contributions to this: tree-level corrections of the form $g g \rightarrow \gamma \gamma+q \bar{q}$ and loop-induced corrections $g g \rightarrow \gamma \gamma$. Phenomenologically, the former are typically very small and we will not discuss them further. The loop-induced contribution is instead quite interesting. First, the large gluon flux at the Large Hadron Collider (LHC) compensates for the $\alpha_{s}$ suppression, making it important for precision phenomenological studies. Being a new channel, it has all the features of a leading order process, in particular large perturbative uncertainties. Moreover, being gluon induced one expects particularly large radiative corrections. This has spurred many investigations, which upgraded the precision in this channel to next-to-leading order $(\mathrm{NLO})$ in QCD, i.e. to $\mathcal{O}\left(\alpha_{s}^{3}\right)[18,19]$.

Given the ever-increasing experimental precision on diphoton measurements [20, 21], it becomes interesting to try and push the theoretical precision even further and consider NNLO corrections to the $g g \rightarrow \gamma \gamma$ process. While this is desirable for a variety of LHC analyses, it is of particular importance for Higgs studies. Indeed, in this case there is a subtle signal/background interference effect between the $g g \rightarrow H \rightarrow \gamma \gamma$ signal and the continuum $g g \rightarrow \gamma \gamma$ background, which is known to modify the Higgs line-shape [22]. This effect can in turn be used to constrain the Higgs boson total decay width [23]. This kind of investigations require an exquisite experimental control, see e.g. ref. [24], as well as robust control of theoretical predictions for both the signal and the background processes. Several in-depth analysis [25-28] suggest that reaching NNLO QCD accuracy in the gluon channel is desirable. A major step towards the calculation of full NNLO QCD corrections to $g g \rightarrow \gamma \gamma$ has been made very recently with the computation of NLO QCD corrections to the $g g \rightarrow \gamma \gamma+j$ process $[10,11]$. In this paper, we present a calculation of the last missing ingredient, the three loop virtual amplitude for the $g g \rightarrow \gamma \gamma$ process.

The rest of this paper is organised as follows. In section 2 we set up our notation and discuss the generic kinematics features of the $g g \rightarrow \gamma \gamma$ process. In section 3 we briefly review the approach of refs $[29,30]$ to the calculation of helicity amplitudes that we adopt here. In section 4 we provide more technical details on our three-loop calculation. In section 5 we discuss the ultraviolet and infrared structure of the scattering amplitude, and define the renormalised finite remainders which are the main result of this paper. In section 6 we document the checks that we have performed on our calculation, and briefly describe the general structure of our result. We also present analytic formulas for the three loop finite remainder for the simplest helicity configuration. The analytic formulas for all the relevant helicity configurations can be found in computer-readable format in the supplementary material that accompany this submission. Finally, we conclude in section 7 .

\section{Notation and kinematics}

We consider virtual QCD corrections to the production of two photons through gluon fusion

$$
g\left(p_{1}\right)+g\left(p_{2}\right) \rightarrow \gamma\left(-p_{3}\right)+\gamma\left(-p_{4}\right)
$$

mediated by light quarks. The signs of the momenta are chosen such that all momenta are incoming, $p_{1}+p_{2}+p_{3}+p_{4}=0$. All particles in the process are on the mass-shell, 
$p_{1}^{2}=p_{2}^{2}=p_{3}^{2}=p_{4}^{2}=0$. The kinematics is fully described by the usual Mandelstam invariants

$$
s=\left(p_{1}+p_{2}\right)^{2}, t=\left(p_{1}+p_{3}\right)^{2}, u=\left(p_{2}+p_{3}\right)^{2}, \quad s+t+u=0 .
$$

In the physical scattering region, one has $s>0, t<0, u<0$. For later reference, we also introduce the dimensionless ratio

$$
x=-\frac{t}{s},
$$

where $0<x<1$ in the physical region. We work in $d=4-2 \epsilon$ dimensions to regulate ultraviolet (UV) and infrared (IR) divergences. To be precise, we adopt the 't Hooft-Veltman (tHV) scheme [31], i.e. we perform computations for generic $d$ but we constrain all the external particles and their polarisations to live in the physical $d=4$ subspace. This allows us to simplify the calculation compared to the Conventional Dimensional Regularisation (CDR) case, where internal and external degrees of freedom are treated as $d$-dimensional.

We write the scattering amplitude for the process in eq. (2.1) as

$$
\begin{aligned}
\mathcal{A}(s, t) & =\delta^{a_{1} a_{2}}(4 \pi \alpha) A(s, t) \\
& =\delta^{a_{1} a_{2}}(4 \pi \alpha) A^{\mu \nu \rho \sigma}(s, t) \epsilon_{1, \mu}\left(p_{1}\right) \epsilon_{2, \nu}\left(p_{2}\right) \epsilon_{3, \rho}\left(p_{3}\right) \epsilon_{4, \sigma}\left(p_{4}\right),
\end{aligned}
$$

where $a_{j}$ is the colour index of the gluon of momentum $p_{j}$ and $\epsilon_{j, \mu}\left(p_{j}\right)$ is the polarisation vector of the vector boson of momentum $p_{j}$. For convenience, we have extracted the leading-order electroweak coupling written in terms of the fine structure constant $\alpha$, where $e=\sqrt{4 \pi \alpha}$ is the unit of electric charge. We are interested in the QCD perturbative expansion of eq. (2.4)

$$
A(s, t)=\frac{\alpha_{s}}{2 \pi}\left[A^{(1)}(s, t)+\frac{\alpha_{s}}{2 \pi} A^{(2)}(s, t)+\left(\frac{\alpha_{s}}{2 \pi}\right)^{2} A^{(3)}(s, t)+\mathcal{O}\left(\alpha_{s}^{3}\right)\right],
$$

where $\alpha_{s}=\alpha_{s}(\mu)$ is the $\overline{\mathrm{MS}}$ renormalized QCD coupling and the superscript indicates the number of loops $L$. We find it convenient to express the result for $A^{(L)}$ in terms of the quadratic Casimir invariants of theory $C_{A}$ and $C_{F}$. They are defined through

$$
T_{i j}^{a} T_{j k}^{a}=C_{F} \delta_{i k}, \quad f^{a c d} f^{b c d}=C_{A} \delta^{a b},
$$

where $f^{a b c}$ and $T_{i j}^{a}$ are the $\mathrm{SU}(3)$ structure constants and the generators in the fundamental representation, respectively. We normalise the generators as

$$
\operatorname{Tr}\left[T^{a} T^{b}\right]=T_{F} \delta^{a_{1} a_{2}}, \quad T_{F}=\frac{1}{2} .
$$

In $\mathrm{QCD}, C_{A}=3$ and $C_{F}=4 / 3$.

The Feynman diagrams for the process eq. (2.1) can be naturally separated according to whether the two photons couple to the same or to two different closed fermion lines. We then introduce the following short hands for the respective electromagnetic coupling structures

$$
\left(n_{f}^{V}\right)^{2}=\left(\sum_{f} Q_{f}\right)^{2}, \quad n_{f}^{V_{2}}=\sum_{f} Q_{f}^{2}
$$


where the sums run over $n_{f}$ light quarks and $Q_{f}$ is their charge in units of $e$, i.e. $Q_{u, c}=2 / 3$, $Q_{d, s, b}=-1 / 3$. For QCD with 5 flavours, the structures in eq. (2.8) evaluate to $\left(n_{f}^{V}\right)^{2}=$ $(1 / 3)^{2}=1 / 9$ and $n_{f}^{V_{2}}=11 / 9$.

\section{The helicity amplitudes}

In this section, we explain how one can efficiently calculate the amplitude in eq. (2.4) for specific helicities. We start by discussing the tensor $A^{\mu \nu \rho \sigma}$. It can be expanded as

$$
A^{\mu \nu \rho \sigma}(s, t)=\sum_{i} \mathcal{F}_{i}(s, t) \Gamma_{i}^{\mu \nu \rho \sigma},
$$

where $\mathcal{F}_{i}$ are scalar form factors ${ }^{1}$ and $\Gamma_{i}^{\mu \nu \rho \sigma}$ are independent tensor structures constructed using external momenta $\left\{p_{i}^{\mu}\right\}$ and the metric tensor $g^{\mu \nu}$. With three independent external momenta, the total number of tensor structures that one can write is 138, see e.g. [32]. Since $A^{\mu \nu \rho \sigma}$ has to be contracted with the external polarisation vectors $\epsilon_{i}^{\mu}$, one can use the physical conditions $p_{i} \cdot \epsilon_{i}=0$ to remove all tensors proportional to $p_{1}^{\mu}, p_{2}^{\nu}, p_{3}^{\rho}, p_{4}^{\sigma}$. This removes all but 57 structures. By making a specific choice for the reference vectors of the external gauge bosons, one may eliminate further redundancies. A convenient choice is to impose

$$
\epsilon_{i} \cdot p_{i+1}=0, \text { where } i=1, \ldots, 4 \text { and } p_{5} \equiv p_{1} .
$$

This leaves one with 10 independent structures, that we choose as

$$
\begin{array}{rlrl}
\Gamma_{1}^{\mu \nu \rho \sigma} & =p_{3}^{\mu} p_{1}^{\nu} p_{1}^{\rho} p_{2}^{\sigma}, & \Gamma_{2}^{\mu \nu \rho \sigma}=p_{3}^{\mu} p_{1}^{\nu} g^{\rho \sigma}, \\
\Gamma_{3}^{\mu \nu \rho \sigma}=p_{3}^{\mu} p_{1}^{\rho} g^{\nu \sigma}, & \Gamma_{4}^{\mu \nu \rho \sigma}=p_{3}^{\mu} p_{2}^{\sigma} g^{\nu \rho}, \\
\Gamma_{5}^{\mu \nu \rho \sigma}=p_{1}^{\nu} p_{1}^{\rho} g^{\mu \sigma}, & \Gamma_{6}^{\mu \nu \rho \sigma}=p_{1}^{\nu} p_{2}^{\sigma} g^{\mu \rho}, \\
\Gamma_{7}^{\mu \nu \rho \sigma}=p_{1}^{\rho} p_{2}^{\sigma} g^{\mu \nu}, & \Gamma_{8}^{\mu \nu \rho \sigma}=g^{\mu \nu} g^{\rho \sigma}, \\
\Gamma_{9}^{\mu \nu \rho \sigma}=g^{\mu \sigma} g^{\nu \rho}, & \Gamma_{10}^{\mu \nu \rho \sigma}=g^{\mu \rho} g^{\nu \sigma} .
\end{array}
$$

For notational convenience, we define the 10 independent structures

$$
T_{i}=\Gamma_{i}^{\mu \nu \rho \sigma} \epsilon_{1, \mu} \epsilon_{2, \nu} \epsilon_{3, \rho} \epsilon_{4, \sigma}
$$

and refer to them, with a slight abuse of language, as tensors. The scattering amplitude eq. (2.4) can then be written as

$$
A(s, t)=\sum_{i=1}^{10} \mathcal{F}_{i}(s, t) T_{i}
$$

We stress that eq. (3.5) is valid at any perturbative order and for any space-time dimension.

In four dimensions, it is easy to see that only 8 out of the 10 tensors $T_{i}$ are actually independent. It turns out that in the tHV scheme, it is possible to separate the purely

\footnotetext{
${ }^{1}$ We note that the form factors $\mathcal{F}_{i}$ also depend on the dimension of the space-time. This dependence is assumed.
} 
four-dimensional tensor structures from the $-2 \epsilon$-dimensional ones through a simple orthogonalisation procedure $[29,30]$. We briefly sketch how this can be done for our process, and refer the reader to refs $[29,30]$ for a thorough discussion. Following ref. [30], we introduce a new tensor basis $\bar{T}_{i}$

$$
A(s, t)=\sum_{i=1}^{10} \overline{\mathcal{F}}_{i}(s, t) \bar{T}_{i},
$$

where the first 7 tensors are identical to the ones introduced before

$$
\bar{T}_{i}=T_{i}, \quad i=1, \ldots, 7
$$

while $\bar{T}_{8}$ is a symmetrised version of $T_{8}$

$$
\bar{T}_{8}=T_{8}+T_{9}+T_{10}
$$

It turns out [30] that these 8 tensors span the physical $d=4$ subspace and do not have any component in the $-2 \epsilon$ directions. The last two tensors $\bar{T}_{9,10}$ can then be chosen in such a way that they are constrained to live in the $-2 \epsilon$ subspace. This can be achieved by simply removing from the original $T_{9,10}$ their projection along $\bar{T}_{1 \ldots 8}$

$$
\bar{T}_{i}=T_{i}-\sum_{j=1}^{8}\left(\mathcal{P}_{j} T_{i}\right) \bar{T}_{j}, i=9,10,
$$

where the projectors $\mathcal{P}_{i}$ are defined through

$$
\sum_{\mathrm{pol}} \mathcal{P}_{i} \bar{T}_{j}=\delta_{i j}
$$

The explicit form of the $\mathcal{P}_{i}$ projectors relevant for our case can be found in ref. [30]. The new tensors $\bar{T}_{9,10}$ read

$$
\begin{gathered}
\bar{T}_{9}=T_{9}-\frac{1}{3}\left(-\frac{2 \bar{T}_{1}}{s u}-\frac{\bar{T}_{6}}{s}-\frac{\bar{T}_{2}+\bar{T}_{3}+2 \bar{T}_{4}-2 \bar{T}_{5}-\bar{T}_{6}-\bar{T}_{7}}{t}+\frac{\bar{T}_{3}}{u}+\bar{T}_{8}\right), \\
\bar{T}_{10}=T_{10}-\frac{1}{3}\left(\frac{4 \bar{T}_{1}}{s u}+\frac{2 \bar{T}_{6}}{s}-\frac{\bar{T}_{2}-2 \bar{T}_{3}-\bar{T}_{4}+\bar{T}_{5}+2 \bar{T}_{6}-\bar{T}_{7}}{t}-\frac{2 \bar{T}_{3}}{u}+\bar{T}_{8}\right) .
\end{gathered}
$$

The tensors $\bar{T}_{9,10}$ so constructed identically vanish if they are computed using physical polarisation vectors in $d=4$ space-time dimensions and can be safely dropped if one is after tHV helicity amplitudes [30]. For a given helicity configuration we then write

$$
A_{\lambda_{1} \lambda_{2} \lambda_{3} \lambda_{4}}(s, t)=\sum_{i=1}^{8} \overline{\mathcal{F}}_{i}(s, t) \bar{T}_{i, \lambda_{1} \lambda_{2} \lambda_{3} \lambda_{4}}
$$

where $\bar{T}_{i, \lambda_{1} \lambda_{2} \lambda_{3} \lambda_{4}}$ are the tensors evaluated with polarisation vectors for well-defined helicity states $\lambda_{i}$. It should not be surprising that the generic helicity amplitude can be parametrised in terms of 8 independent structures. Indeed, in four dimensions we would 
need to consider $2^{4}=16$ independent helicity amplitudes. However, half of them can be related by parity, which leaves us with 8 independent helicity states. These are in one-to-one correspondence with the 8 form factors $\overline{\mathcal{F}}_{i}$.

When dealing with helicity amplitudes, we find it convenient to factor out a spinor function carrying the relevant helicity weight. We achieve this by writing

$$
A_{\lambda_{1} \lambda_{2} \lambda_{3} \lambda_{4}}(s, t)=\mathcal{S}_{\lambda_{1} \lambda_{2} \lambda_{3} \lambda_{4}} f_{\lambda_{1} \lambda_{2} \lambda_{3} \lambda_{4}}(s, t),
$$

where

$$
\begin{aligned}
& \mathcal{S}_{++++}=\frac{[12][34]}{\langle 12\rangle\langle 34\rangle}, \\
& \mathcal{S}_{-+++}=\frac{\langle 12\rangle\langle 14\rangle[24]}{\langle 34\rangle\langle 23\rangle\langle 24\rangle}, \\
& \mathcal{S}_{+-++}=\frac{\langle 21\rangle\langle 24\rangle[14]}{\langle 34\rangle\langle 13\rangle\langle 14\rangle}, \\
& \mathcal{S}_{++-+}=\frac{\langle 32\rangle\langle 34\rangle[24]}{\langle 14\rangle\langle 21\rangle\langle 24\rangle}, \\
& \mathcal{S}_{+++-}=\frac{\langle 42\rangle\langle 43\rangle[23]}{\langle 13\rangle\langle 21\rangle\langle 23\rangle}, \\
& \mathcal{S}_{--++}=\frac{\langle 12\rangle[34]}{[12]\langle 34\rangle}, \\
& \mathcal{S}_{-+-+}=\frac{\langle 13\rangle[24]}{[13]\langle 24\rangle}, \\
& \mathcal{S}_{+--+}=\frac{\langle 23\rangle[14]}{[23]\langle 14\rangle},
\end{aligned}
$$

and

$$
\begin{aligned}
& f_{++++}=\frac{t^{2}}{4}\left(\frac{2 \overline{\mathcal{F}}_{6}}{u}-\frac{2 \overline{\mathcal{F}}_{3}}{s}-\overline{\mathcal{F}}_{1}\right)+\overline{\mathcal{F}}_{8}\left(\frac{s}{u}+\frac{u}{s}+4\right)+\frac{t}{2}\left(\overline{\mathcal{F}}_{2}-\overline{\mathcal{F}}_{4}+\overline{\mathcal{F}}_{5}-\overline{\mathcal{F}}_{7}\right), \\
& f_{-+++}=\frac{t^{2}}{4}\left(\frac{2 \overline{\mathcal{F}}_{3}}{s}+\overline{\mathcal{F}}_{1}\right)+t\left(\frac{\overline{\mathcal{F}}_{8}}{s}+\frac{1}{2}\left(\overline{\mathcal{F}}_{4}+\overline{\mathcal{F}}_{6}-\overline{\mathcal{F}}_{2}\right)\right) \\
& f_{+-++}=-\frac{t^{2}}{4}\left(\frac{2 \overline{\mathcal{F}}_{6}}{u}-\overline{\mathcal{F}}_{1}\right)+t\left(\frac{\overline{\mathcal{F}}_{8}}{u}-\frac{1}{2}\left(\overline{\mathcal{F}}_{2}+\overline{\mathcal{F}}_{3}+\overline{\mathcal{F}}_{5}\right)\right) \\
& f_{++-+}=\frac{t^{2}}{4}\left(\frac{2 \overline{\mathcal{F}}_{3}}{s}+\overline{\mathcal{F}}_{1}\right)+t\left(\frac{\overline{\mathcal{F}}_{8}}{s}+\frac{1}{2}\left(\overline{\mathcal{F}}_{6}+\overline{\mathcal{F}}_{7}-\overline{\mathcal{F}}_{5}\right)\right) \\
& f_{+++-}=-\frac{t^{2}}{4}\left(\frac{2 \overline{\mathcal{F}}_{6}}{u}-\overline{\mathcal{F}}_{1}\right)+t\left(\frac{\overline{\mathcal{F}}_{8}}{u}+\frac{1}{2}\left(\overline{\mathcal{F}}_{4}+\overline{\mathcal{F}}_{7}-\overline{\mathcal{F}}_{3}\right)\right) \\
& f_{--++}=-\frac{t^{2}}{4} \overline{\mathcal{F}}_{1}+\frac{1}{2} t\left(\overline{\mathcal{F}}_{2}+\overline{\mathcal{F}}_{3}-\overline{\mathcal{F}}_{6}-\overline{\mathcal{F}}_{7}\right)+2 \overline{\mathcal{F}}_{8} \\
& f_{-+-+}=t^{2}\left(\frac{\overline{\mathcal{F}}_{8}}{s u}-\frac{\overline{\mathcal{F}}_{3}}{2 s}+\frac{\overline{\mathcal{F}}_{6}}{2 u}-\frac{\overline{\mathcal{F}}_{1}}{4}\right) \\
& f_{+--+}=-\frac{t^{2}}{4} \overline{\mathcal{F}}_{1}+\frac{1}{2} t\left(\overline{\mathcal{F}}_{3}-\overline{\mathcal{F}}_{4}+\overline{\mathcal{F}}_{5}-\overline{\mathcal{F}}_{6}\right)+2 \overline{\mathcal{F}}_{8} .
\end{aligned}
$$

We note that we have chosen the spinor functions in eq. (3.14) following ref. [18]. The expressions for the spinor-free amplitudes $f_{\lambda_{1} \lambda_{2} \lambda_{3} \lambda_{4}}$ can be easily obtained by computing the relevant $\bar{T}_{i}$ with polarisation vectors for fixed helicity states. We also note that we define " $\pm "$ helicity states as ${ }^{2}$

$$
\epsilon_{j,-}^{\mu}\left(p_{j}\right)=\frac{\left\langle p_{j}\left|\gamma^{\mu}\right| q_{j}\right]}{\sqrt{2}\left[p_{j} q_{j}\right]}, \quad \epsilon_{j,+}^{\mu}\left(p_{j}\right)=\frac{\left\langle q_{j}\left|\gamma^{\mu}\right| p_{j}\right]}{\sqrt{2}\left\langle q_{j} p_{j}\right\rangle},
$$

\footnotetext{
${ }^{2}$ See e.g. ref. [33] for a review of the spinor-helicity formalism. We follow the notation of [33], with the identification $\left.\left|i^{+}\right\rangle=|i\rangle,\left|i^{-}\right\rangle=\mid i\right],\left\langle i^{+}\right|=\left[i \mid,\left\langle i^{-}\right|=\langle i|\right.$, and complex conjugation $\langle i j\rangle^{*}=[j i]$.
} 
where $q_{j}$ is the reference vector for the boson $j$, irrespective of whether the particles are in the initial or the final state.

We have written eqs. (3.14), (3.15) for only 8 helicity states. The 8 remaining ones can be obtained from these by exploiting parity invariance,

$$
A_{\lambda_{1} \lambda_{2} \lambda_{3} \lambda_{4}}=A_{-\lambda_{1},-\lambda_{2},-\lambda_{3},-\lambda_{4}}(\langle i j\rangle \leftrightarrow[j i])
$$

where $-\lambda_{i}$ indicates the opposite helicity of $\lambda_{i}$. We also note that the helicity amplitudes must obey Bose symmetry, i.e. they must be symmetric under the exchange of $1 \leftrightarrow 2$ and/or $3 \leftrightarrow 4$. In terms of the spinor-free amplitudes, this implies

$$
\begin{aligned}
& f_{\lambda_{2} \lambda_{1} \lambda_{3} \lambda_{4}}(s, t)=f_{\lambda_{1} \lambda_{2} \lambda_{3} \lambda_{4}}(s, u), \\
& f_{\lambda_{1} \lambda_{2} \lambda_{4} \lambda_{3}}(s, t)=f_{\lambda_{1} \lambda_{2} \lambda_{3} \lambda_{4}}(s, u),
\end{aligned}
$$

with $u=-s-t$. These relations provide non-trivial checks for our results.

\section{Details of the calculation}

The spinor-free helicity amplitudes $f_{\lambda_{1} \lambda_{2} \lambda_{3} \lambda_{4}}$ can be computed as perturbative series in the QCD coupling constant $\alpha_{s}$. For a generic helicity configuration we introduce the short hand $\vec{\lambda}=\left(\lambda_{1}, \lambda_{2}, \lambda_{3}, \lambda_{4}\right)$ and write

$$
f_{\vec{\lambda}}=\sum_{L=1}^{3}\left(\frac{\alpha_{s, b}}{2 \pi}\right)^{L} f_{\vec{\lambda}}^{(L, b)}+\mathcal{O}\left(\alpha_{s, b}^{4}\right),
$$

where $\alpha_{s, b}$ is the bare strong coupling constant and $f_{\vec{\lambda}}^{(L, b)}$ is the bare perturbative coefficient of the helicity amplitude. Since the leading order contribution $f_{\vec{\lambda}}^{(1, b)}$ to the production of two photons in gluon fusion already involves one-loop integrals, the next-to-next-to-leading order contribution $f_{\vec{\lambda}}^{(3, b)}$ involves three-loop integrals. The main goal of this paper is to calculate $f_{\vec{\lambda}}^{(3, b)}$.

As explained in section 3 , we can obtain the helicity amplitudes by computing the $\overline{\mathcal{F}}_{i}$, $i=1, \ldots, 8$ form factors. In principle, this can be achieved straightforwardly by applying the projectors $\mathcal{P}_{i}, i=1, \ldots, 8$, of section 3 to the sum of all the relevant Feynman diagrams. At three loops, this leads to a sum of terms of the form

$$
\int\left(\prod_{i=1}^{3} \mathcal{D}^{d} k_{i}\right) \frac{\mathcal{N}\left(d ;\left\{p_{i} \cdot p_{j}\right\},\left\{p_{i} \cdot k_{j}\right\},\left\{k_{i} \cdot k_{j}\right\}\right)}{D_{1}^{n_{1}} \ldots D_{10}^{n_{10}}},
$$

where $k_{i}, i=1,2,3$, are the loop momenta, $D_{i}$ are the propagators of the graphs and $n_{i}$ are non-negative integers. Following previous work [34, 35], the integration measure for every loop is defined as

$$
\int \mathcal{D}^{d} k_{i}=e^{\epsilon \gamma_{E}} \int \frac{d^{d} k_{i}}{i \pi^{d / 2}}
$$

It is convenient to treat propagators and scalar products involving the loop momenta on the same footings. We do this by writing scalar products in the numerator as additional 


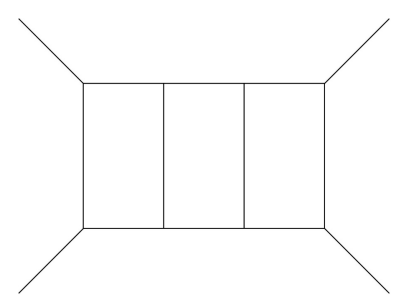

$(\mathrm{PL})$.

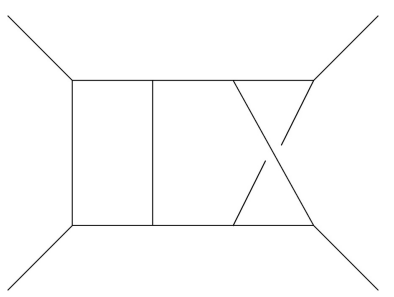

(NPL1).

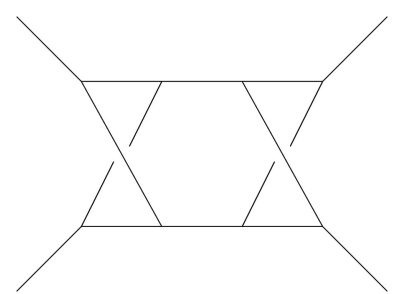

(NPL2).

Figure 1. Representative top level topologies for the planar (PL), single nonplanar (NPL1), and double nonplanar (NPL2) integral families.

propagators raised to negative powers. For our problem, there are 6 scalar products of the form $k_{i} \cdot k_{j}$ and 9 of the form $k_{i} \cdot p_{j}$, so we can write a generic Feynman integral of the form eq. (4.2) as

$$
\int\left(\prod_{i=1}^{3} \mathcal{D}^{d} k_{i}\right) \frac{f\left(d ;\left\{p_{i} \cdot p_{j}\right\}\right)}{D_{1}^{n_{1}} \ldots D_{15}^{n_{15}}},
$$

where now $n_{i}$ can also be negative integers. We refer to each set of inequivalent $\left\{D_{1}, \ldots, D_{15}\right\}$ as an "integral family". Within each family, it is well known that not all the integrals are linearly independent. Indeed, Feynman integrals satisfy integration-by-parts (IBP) identities [36] of the form

$$
\int\left(\prod_{i=1}^{3} \mathcal{D}^{d} k_{i}\right) \frac{\partial}{\partial k_{j}^{\mu}} \frac{v_{j}^{\mu}}{D_{1}^{n_{1}} \ldots D_{m}^{n_{m}}}=0
$$

where $v_{j}$ can be any loop or external momentum. In principle, it is possible to use these identities to express all the $\overline{\mathcal{F}}_{i}$ form factors in terms of a minimal set of independent "master integrals" (MI) [37]. While all the steps described above are well-understood in principle, the complexity involved in intermediate stages grows very quickly with the number of loops and external scales. In our case, the three-loop calculation involves 3 different families, each of which can contribute with 6 independent crossings of the external legs, and more than $4 \times 10^{6}$ integrals to the amplitude. Moreover, using (4.5) directly would lead to a very large number of equations involving also many additional auxiliary integrals. We now describe the procedure that we have adopted to keep the degree of complexity manageable.

First, we generated all Feynman diagrams with Qgraf [38] and mapped each diagram to an integral family using Reduze $2[39,40]$ to generate the required shifts of loop momenta. At this stage, it is useful to group diagrams that present similar structures together and perform the $\mathcal{P}_{1, \ldots, 8}$ projections for each of these groups separately. This can be done by keeping together diagrams that can be mapped to the same crossing of the same integral families. This allows us to reduce redundancy in the algebraic manipulations required. Examples of top sectors from our three families of integrals are depicted in figure 1, while their complete definition can be found in the supplementary material. To evaluate the contributions to the form factors, we performed the colour, Lorentz and Dirac algebra as well as further symbolic manipulations described in the following with Form [41]. 
We find it important to stress that by expressing the result for each $\overline{\mathcal{F}}_{1, \ldots, 8}$ in terms of a minimal set of integrals under crossings and shift symmetries, prior to performing the actual IBP reduction, we saw a significant decrease in complexity. This is expected, as many equivalent integrals are combined together and redundant structures are removed. After this simplification, we used the $\overline{\mathcal{F}}_{i}$ to construct the spinor-free helicity amplitudes, and collected the contributions to different colour structures. This way, we arrived at a minimal set of gauge-independent building blocks. We found it useful to partial fraction the rational functions with respect to $x$ in order to reduce their complexity.

The next step is the actual IBP integral reduction. We did this using an in-house implementation of the Laporta algorithm [37], Finred [42], which exploits syzygy-based techniques [43-48] and finite-field arithmetics [42, 49-51]. We found in this way that the three loop helicity amplitudes can be expressed in terms of the 221 MIs computed in ref. [34] and crossed versions of them, for a total of 486 MIs. $^{3}$ We stress that these MIs are pure functions, i.e. they do not have any non-trivial rational functions of $x$ or $d$ as prefactors. Before inserting the IBP relations into the amplitude, we partial fractioned them with respect to both $d$ and $x$. We found that this step is crucial to keep the complexity under control. Finally, we performed one last partial fraction decomposition of the full amplitude after we wrote it in terms of MIs.

As a last step, we expand in $\epsilon$ and substitute the analytic results for the MIs. All of the integrals required for our calculation were computed in ref. [34]. Their $\epsilon$ expansion can be written in terms of Harmonic Polylogarithms (HPL), that we define iteratively as ${ }^{4}$

$$
G(\underbrace{0, \ldots, 0}_{n \text { times }} ; x) \equiv \frac{\ln ^{n} x}{n !}, \quad G\left(a_{n}, \ldots, a_{1} ; x\right)=\int_{0}^{x} \frac{d z}{z-a_{n}} G\left(a_{n-1}, \ldots, a_{1} ; z\right),
$$

with $G(x)=1$ and $a_{i} \in\{0,1\}$. For our case, we need to consider polylogarithms up to weight 6 , i.e. $n=6$ in eq. (4.6). We used the Mathematica package PolyLogTools [54] to manipulate HPLs up to weight 5, augmented by a straightforward generalisation of its routines up to the required weight 6 , as well as an independent package for multiple polylogarithms written by one of us. As expected from the fact that there are fewer weight $\leq 6$ HPLs than MIs, we observed a noticeable decrease in complexity for the amplitude after we expressed it in term of HPLs. We summarise the degree of complexity of the various steps discussed above in table 1.

Before presenting our results, we note that although the MIs have been computed in ref. [34], for this calculation we have decided to recompute them as an independent check. We used the same definitions for the MIs as ref. [34], and followed the same strategy outlined in that reference for obtaining their analytic. First, since the basis [34] is pure and of uniform weight [55] the MIs obey very simple differential equations

$$
\mathrm{d} \vec{M}(\epsilon ; s, t, u)=\epsilon\left[A_{s} \mathrm{~d} \log (s)+A_{t} \mathrm{~d} \log (t)+A_{u} \mathrm{~d} \log (u)\right] \vec{M}(\epsilon ; s, t, u),
$$

\footnotetext{
${ }^{3}$ We note that while the reductions of some integrals were already known from earlier calculations [3, 35], for this process we had to reduce a significant number of new integrals compared to those references.

${ }^{4}$ Note that we use the GPL notation of ref. [52], rather than the original HPL notation of ref. [53].
} 


\begin{tabular}{|l|l|l|l|}
\hline & $1 \mathrm{~L}$ & $2 \mathrm{~L}$ & $3 \mathrm{~L}$ \\
\hline Number of diagrams & 6 & 138 & 3299 \\
\hline Number of inequivalent integral families & 1 & 2 & 3 \\
\hline Number of integrals before IBPs and symmetries & 209 & 20935 & 4370070 \\
\hline Number of master integrals & 6 & 39 & 486 \\
\hline Size of the Qgraf result [kB] & 4 & 90 & 2820 \\
\hline Size of the Form result before IBPs and symmetries [kB] & 276 & 54364 & 19734644 \\
\hline Size of helicity amplitudes written in terms of MIs [kB] & 12 & 562 & 304409 \\
\hline Size of helicity amplitudes written in terms of HPLs [kB] & 136 & 380 & 1195 \\
\hline
\end{tabular}

Table 1. Complexity of the various stages of the calculation at different loop orders.

where $\vec{M}$ is a vector whose components are the MIs and $A_{i}$ are constant matrices. Using the basis of ref. [34] we have rederived the differential equation from scratch and found agreement. Given the simple form of eq. (4.7), it is straightforward to iteratively solve it order by order in $\epsilon$, modulo boundary conditions. The only non-trivial issue is how to fix the latter. Very interestingly, the authors of ref. [34] noted that at three loops it is enough to impose regularity conditions to fix all boundary conditions, apart from one simple overall normalisation. The main idea is to look at the differential equation near singular points $s \rightarrow 0, t \rightarrow 0, u \rightarrow 0$. Let us consider $s \rightarrow 0$ as an example. In this limit the general solution of eq. (4.7) behaves like

$$
\vec{M} \sim s^{A_{s} \epsilon} \vec{M}_{0, s}
$$

where $\vec{M}_{0, s}$ is a constant vector. It was argued in ref. [34] that the MIs considered here can only develop branch cuts of the form $s^{-\alpha \epsilon}$ with $\alpha>0$. This implies that the coefficient of $s^{\alpha \epsilon}$ in $s^{A_{s} \epsilon} \vec{M}_{0, s}$ must vanish for $\alpha>0$. As a consequence, there must exist non-trivial relations between different MIs in the $s \rightarrow 0$ limit. When combined with analogous relations derived from the limits $t, u \rightarrow 0$, the authors of ref. [34] found that for the case under study one can completely constrain all the boundary conditions up to an overall normalisation factor. We have independently verified that this is the case, which allowed us to rederive an analytic expression for all the master integrals. We have then verified that our results to weight 6 are identical to the ones of ref. [34], provided that the latter are analytically continued to the physical Riemann sheet. Since in ref. [34] final results are only presented for one single crossing, for convenience we decided to provide analytic results for all the three-loop master integrals and all their crossings in the supplementary material accompanying this publication. We also provide weight 6 results for a uniform-weight basis of the two-loop integrals.

\section{UV renormalisation and IR regularisation}

Following the steps outlined above, we obtained analytical expressions for the bare helicity amplitudes $f_{\vec{\lambda}}^{(L, b)}$ defined in eq. (4.1) for $L=1,2,3$. The $f_{\vec{\lambda}}^{(L, b)}$ are affected by both ultraviolet (UV) and infrared (IR) divergences, which manifest themselves as poles in the 
dimensional regularisation parameter $\epsilon=(4-d) / 2$. While the former are removed by UV renormalisation, the latter can be regularised using universal IR operators acting on lower-loop amplitudes. We now discuss in detail how this can be done.

We first consider UV divergences. We define $\alpha_{s}(\mu)$ to be the renormalised strong coupling constant in the $\overline{\mathrm{MS}}$ scheme at the scale $\mu$

$$
S_{\epsilon} \mu_{0}^{2 \epsilon} \alpha_{s, b}=\mu^{2 \epsilon} \alpha_{s}(\mu) Z\left[\alpha_{s}(\mu)\right]
$$

with $S_{\epsilon}=(4 \pi)^{\epsilon} e^{-\gamma_{E} \epsilon}$ and

$$
Z[\alpha]=1-\frac{\beta_{0}}{\epsilon}\left(\frac{\alpha_{s}}{2 \pi}\right)+\left(\frac{\beta_{0}^{2}}{\epsilon^{2}}-\frac{\beta_{1}}{2 \epsilon}\right)\left(\frac{\alpha_{s}}{2 \pi}\right)^{2}+\mathcal{O}\left(\alpha_{s}^{3}\right) .
$$

The first two coefficients of the QCD beta function read

$$
\beta_{0}=\frac{11}{6} C_{A}-\frac{2}{3} T_{F} n_{f}, \quad \beta_{1}=\frac{17}{6} C_{A}^{2}-T_{F} n_{f}\left(\frac{5}{3} C_{A}+C_{F}\right) .
$$

We then expand the spinor-free helicity amplitudes $f_{\vec{\lambda}}$ in terms of the renormalised strong coupling $\alpha_{s}(\mu)$ as

$$
f_{\vec{\lambda}}=\sum_{L=1}^{3}\left(\frac{\alpha_{s}(\mu)}{2 \pi}\right)^{L} f_{\vec{\lambda}}^{(L)} .
$$

The expression for the renormalized amplitudes $f_{\vec{\lambda}}^{(L)}$ can be obtained by substituting eq. (5.1) in eq. (4.1) and expanding in the renormalised coupling. For convenience, we will set $\mu^{2}=s$ in the following. The result for arbitrary scale can be easily obtained using renormalisation group methods.

We now consider IR divergences. The IR structure of the amplitude is governed by the soft and collinear behaviour of virtual quarks and gluons and it is universal, i.e. it only depends on the colour and nature of the external legs. This allows one to write the renormalised amplitude as

$$
\begin{aligned}
& f_{\vec{\lambda}}^{(1)}=f_{\vec{\lambda}}^{(1, \text { fin })} \\
& f_{\vec{\lambda}}^{(2)}=\mathcal{I}_{1} f_{\vec{\lambda}}^{(1)}+f_{\vec{\lambda}}^{(2, \text { fin })}, \\
& f_{\vec{\lambda}}^{(3)}=\mathcal{I}_{2} f_{\vec{\lambda}}^{(1)}+\mathcal{I}_{1} f_{\vec{\lambda}}^{(2)}+f_{\vec{\lambda}}^{(3, \text { fin })}
\end{aligned}
$$

where $f_{\vec{\lambda}}^{(i, \text { fin) }}$ are finite in four dimensions. The IR structure is encoded in the operators $\mathcal{I}_{i}$, that for our case (with $\mu^{2}=s$ ) read [56]

$$
\begin{aligned}
& \mathcal{I}_{1}(\epsilon)=-\frac{e^{i \pi \epsilon} e^{\gamma_{E} \epsilon}}{\Gamma(1-\epsilon)}\left(\frac{C_{A}}{\epsilon^{2}}+\frac{\beta_{0}}{\epsilon}\right) \\
& \mathcal{I}_{2}(\epsilon)=-\frac{1}{2} \mathcal{I}_{1}(\epsilon)\left(\mathcal{I}_{1}(\epsilon)+\frac{2 \beta_{0}}{\epsilon}\right)+\frac{e^{-\gamma_{E} \epsilon} \Gamma(1-2 \epsilon)}{\Gamma(1-\epsilon)}\left(\frac{\beta_{0}}{\epsilon}+K\right) \mathcal{I}_{1}(2 \epsilon)+2 \frac{e^{\epsilon \gamma_{E}}}{\Gamma(1-\epsilon)} H_{g},
\end{aligned}
$$

where $K$ is the next-to-leading-order coefficient of the cusp anomalous dimension

$$
K=\left(\frac{67}{18}-\frac{\pi^{2}}{6}\right) C_{A}-\frac{10}{9} n_{f} T_{F}
$$


and $[57]$

$$
H_{g}=\frac{1}{2 \epsilon}\left[\left(\frac{\zeta(3)}{4}+\frac{5}{24}+\frac{11 \pi^{2}}{288}\right) C_{A}^{2}+T_{F} n_{f}\left(\frac{C_{F}}{2}-\left(\frac{29}{27}+\frac{\pi^{2}}{72}\right) C_{A}\right)+\frac{10}{27} T_{F}^{2} n_{f}^{2}\right] .
$$

In eqs (5.5) we used the fact that diphoton production in gluon fusion starts at one loop. The finite remainders for the helicity amplitudes $f_{\vec{\lambda}}^{(L, f i n)}$ up to three loops are the main result of this paper, and we provide analytic results for them in the supplementary material.

\section{Checks and structure of the result}

We have performed various checks on the correctness of our results. First, we have employed two derivations of the three-loop $\mathcal{F}_{i}$ form factors at the integrand level and verified that they agree. We have also compared the one- and two-loop helicity amplitudes against the results of ref. [18] and found agreement. To validate our numerical evaluation procedure, we also checked the helicity-summed one-loop squared amplitude against OpenLoops [58, 59], and one helicity configuration at two loops against MCFM [60,61]. Finally, we have verified that the UV and IR poles up to three loops follow the structure described in the previous section. This provides a strong check of the correctness of the three-loop amplitudes.

We now discuss the general structure of our result. The amplitude can be expressed in terms of the two quadratic Casimirs $C_{A}$ and $C_{F}$ and the flavour structures $n_{f}, n_{f}^{V}$ and $n_{f}^{V_{2}}$ defined in eq. (2.8). At $L$ loops the amplitude is a homogeneous degree- $L$ polynomial in these 5 variables. At one-loop, the amplitude is only proportional to $n_{f}^{V_{2}}$, since the two photons must both couple to the same fermion line. At two loops, the structures $n_{f}^{V_{2}} \times\left\{C_{F}, C_{A}\right\}$ appear in the bare amplitude. The finite remainder contains in addition a term proportional to $n_{f}^{V_{2}} n_{f}$ stemming from $\beta_{0}$ in the UV/IR regularisation. We note that there is no $\left(n_{f}^{V}\right)^{2}$ contribution. It is easy to understand why this is the case. The $\left(n_{f}^{V}\right)^{2}$ colour factor only appears if the two photons are attached to two different (closed) fermion lines. Such diagrams do appear at two loops, but they are of the form of two $\gamma g g^{*}$ one-loop triangles connected through a gluon propagator. Due to an argument analogous to Furry's theorem, these diagrams give no net contribution to the amplitude. A similar argument allows one to conclude that there is no net contribution from Feynman diagrams with colour factors $n_{f}\left(n_{f}^{V}\right)^{2}$ at three loops. Furthermore, it is easy to see that the structure $n_{f}^{2} n_{f}^{V_{2}}$ is absent in the three-loop bare amplitude. ${ }^{5}$ Since there is no $\left(n_{f}^{V}\right)^{2}$ contribution at lower loops, the $\left(n_{f}^{V}\right)^{2}$ term in the bare three loop amplitude must be finite. We observe, however, that it is non-zero. Indeed, at three loops this colour factor appears in triple-box diagrams for which the Furry argument outlined above is no longer applicable.

We now move to the discussion of the kinematic features of the three-loop amplitude, i.e. its $x$ dependence. The amplitude contains terms of the form $G\left(a_{1}, \ldots, a_{n} ; x\right) / x^{k}$ $(-2 \leq k \leq 2)$ and $G\left(a_{1}, \ldots, a_{n} ; x\right) /(1-x)^{k}(1 \leq k \leq 2)$, where $a_{i} \in\{0,1\}, 0 \leq n \leq 6$, and $G$ are the Harmonic Polylogarithms defined in eq. (4.6). Instead of the HPLs, we

\footnotetext{
${ }^{5}$ We note however that the $n_{f}^{2} n_{f}^{V_{2}}$ structure contributes to our finite remainders, since it is induced by the $n_{f}$ dependence of the UV/IR counterterms.
} 
found it useful to also consider the alternative functional basis described in ref. [35] to speed up the numerical evaluation of the final result. Using the algorithm of [62], we have constructed a basis of logarithms, classical polylogarithms and multiple polylogarithms to rewrite the HPLs without introducing any new spurious singularities. We used products of lower weight functions whenever possible and preferred functions whose series representation requires a small number of nested sums. In this way, we found that 23 independent transcendental functions and products thereof suffice to represent our HPLs up to weight 6 . The new basis consists of 2 logarithms, $\ln (x) \ln (1-x), 12$ classical polylogarithms, $\operatorname{Li}_{2}$ of $x$, $\mathrm{Li}_{3}$ of $x$ and $1-x$ and $\mathrm{Li}_{4}, \mathrm{Li}_{5}, \mathrm{Li}_{6}$ of $x, 1-x$ and $-x /(1-x)$, as well as 9 multiple polylogarithms $\operatorname{Li}_{3,2}(1, x), \operatorname{Li}_{3,2}(1-x, 1), \operatorname{Li}_{3,2}(x, 1), \operatorname{Li}_{3,3}(1-x, 1), \operatorname{Li}_{3,3}(x, 1), \operatorname{Li}_{3,3}\left(\frac{-x}{1-x}, 1\right), \operatorname{Li}_{4,2}(1-$ $x, 1), \operatorname{Li}_{4,2}(x, 1), \operatorname{Li}_{2,2,2}(x, 1,1)$. Here, we follow the conventions of ref. [52] and define

$$
\operatorname{Li}_{m_{1}, \ldots, m_{k}}\left(x_{1}, \ldots, x_{k}\right)=\sum_{i_{1}>\ldots>i_{k}>0} \frac{x_{1}^{i_{1}}}{i_{1}^{m_{1}}} \ldots \frac{x_{k}^{i_{k}}}{i_{k}^{m_{k}}} .
$$

In the supplementary material, we provide our analytic results written both in terms of HPLs and in terms of this minimal set of functions. For convenience, we also provide results for the finite remainders of the one- and two-loop helicity amplitudes up to weight 6 .

Finally, we present our results. Although intermediate expressions are rather complicated, see table 1 , we find that the final results are remarkably compact. The $\vec{\lambda}=(++++)$ helicity configuration is particularly simple. This is of course expected, since the one-loop amplitude does not have support on any cut, hence it is purely rational rather than a weight-2 function. This simplicity persists at higher loops. For illustration, we now report here the result for the finite reminders defined in eq. (5.5) up to three loops for this helicity configuration. At one and two loops one has

$$
\begin{aligned}
& f_{++++}^{(1, \text { fin })}=2 n_{f}^{V_{2}}, \\
& f_{++++}^{(2, \text { fin })}=2 n_{f}^{V_{2}}\left(2 C_{A}-3 C_{F}+i \pi \beta_{0}\right) .
\end{aligned}
$$

At three loops, we write the finite remainder as

$$
\begin{aligned}
f_{++++}^{(3, \text { fin })}= & \Delta_{1}(x) n_{f}^{V_{2}} C_{A}^{2}+\Delta_{2}(x) n_{f}^{V_{2}} C_{A} C_{F}+\Delta_{3}(x) n_{f} n_{f}^{V_{2}} C_{A}+\Delta_{4}(x)\left(n_{f}^{V}\right)^{2} C_{A} \\
& +\Delta_{5}(x) n_{f}^{V_{2}} C_{F}^{2}+\Delta_{6}(x)\left(n_{f}^{V}\right)^{2} C_{F}+\Delta_{7}(x) n_{f} n_{f}^{V_{2}} C_{F}+\Delta_{8}(x) n_{f}^{2} n_{f}^{V_{2}} \\
& +\{(x) \leftrightarrow(1-x)\}
\end{aligned}
$$

with

$$
\begin{aligned}
\Delta_{1}(x)= & -\frac{23 L_{1}\left(L_{1}+2 i \pi\right)}{9 x^{2}}+\frac{32 L_{1}\left(L_{1}+2 i \pi\right)-46\left(L_{1}+i \pi\right)}{9 x}-\frac{17}{36} L_{0}^{2}-\frac{19}{36} L_{0} L_{1}+\frac{1}{9} L_{0}-2 i \pi L_{0} \\
& +\frac{1}{288} \pi^{4}-\frac{373}{72} \zeta_{3}-\frac{185}{72} \pi^{2}+\frac{4519}{324}+\frac{1}{2} i \pi \zeta_{3}+\frac{11}{144} i \pi^{3}+\frac{157}{12} i \pi+\frac{43}{9} L_{0} x \\
& -\frac{7}{9} x^{2}\left(\left(L_{0}-L_{1}\right)^{2}+\pi^{2}\right), \\
\Delta_{2}(x)= & \frac{8 L_{1}\left(L_{1}+2 i \pi\right)}{3 x^{2}}+\frac{16\left(L_{1}+i \pi\right)-8 L_{1}\left(L_{1}+2 i \pi\right)}{3 x}-\frac{1}{3} L_{0}^{2}+\frac{5}{6} L_{0} L_{1}+\frac{17}{3} L_{0}+i \pi L_{0}-\frac{5}{12} \pi^{2} \\
& -\frac{199}{6}-8 i \pi-\frac{16}{3} L_{0} x+\frac{4}{3} x^{2}\left(\left(L_{0}-L_{1}\right)^{2}+\pi^{2}\right),
\end{aligned}
$$




$$
\begin{aligned}
\Delta_{3}(x)= & \frac{L_{1}\left(L_{1}+2 i \pi\right)}{18 x^{2}}+\frac{2\left(L_{1}+i \pi\right)-L_{1}\left(L_{1}+2 i \pi\right)}{18 x}-\frac{1}{36} L_{0}^{2}+\frac{1}{36} L_{0} L_{1}-\frac{1}{9} L_{0}-\frac{61}{36} \zeta_{3}+\frac{475}{432} \pi^{2} \\
& -\frac{925}{324}-\frac{1}{72} i \pi^{3}-\frac{175}{54} i \pi+\frac{2}{9} L_{0} x+\frac{1}{36} x^{2}\left(\left(L_{0}-L_{1}\right)^{2}+\pi^{2}\right), \\
\Delta_{4}(x)= & -\frac{5 L_{1}\left(L_{1}+2 i \pi\right)}{4 x^{2}}+\frac{L_{1}\left(L_{1}+2 i \pi\right)-8\left(L_{1}+i \pi\right)}{2 x}+\frac{1}{4} L_{0}^{2}-\frac{1}{4} L_{0} L_{1}-2 L_{0}-6 \zeta_{3}+\frac{1}{8} \pi^{2}-\frac{1}{2} \\
& +4 L_{0} x-x^{2}\left(\left(L_{0}-L_{1}\right)^{2}+\pi^{2}\right), \\
\Delta_{5}(x)= & -\frac{L_{1}\left(L_{1}+2 i \pi\right)}{x^{2}}+\frac{L_{1}\left(L_{1}+2 i \pi\right)-2\left(L_{1}+i \pi\right)}{x}-\frac{1}{2} L_{0}^{2}-i \pi L_{0}+\frac{39}{4}+i \pi+2 L_{0} x \\
& -\frac{1}{2} x^{2}\left(\left(L_{0}-L_{1}\right)^{2}+\pi^{2}\right), \quad 3 x \\
\Delta_{6}(x)= & \frac{10 L_{1}\left(L_{1}+2 i \pi\right)}{3 x^{2}}+\frac{32\left(L_{1}+i \pi\right)-4 L_{1}\left(L_{1}+2 i \pi\right)}{3 x}-\frac{2}{3} L_{0}^{2}+\frac{2}{3} L_{0} L_{1}+\frac{16}{3} L_{0}+16 \zeta_{3}-\frac{1}{3} \pi^{2} \\
& +\frac{4}{3}-\frac{32}{3} L_{0} x+\frac{8}{3} x^{2}\left(\left(L_{0}-L_{1}\right)^{2}+\pi^{2}\right), \\
\Delta_{7}(x)= & \frac{5 L_{1}\left(L_{1}+2 i \pi\right)}{3 x^{2}}+\frac{10\left(L_{1}+i \pi\right)-8 L_{1}\left(L_{1}+2 i \pi\right)}{3 x}+\frac{2}{3} L_{0}^{2}+\frac{1}{3} L_{0} L_{1}-\frac{10}{3} L_{0}+2 i \pi L_{0}+4 \zeta_{3} \\
& -\frac{\pi^{2}}{6}+5-3 i \pi-\frac{10}{3} L_{0} x+\frac{1}{3} x^{2}\left(\left(L_{0}-L_{1}\right)^{2}+\pi^{2}\right), \\
\Delta_{8}(x)= & -\frac{23}{216} \pi^{2}+\frac{5}{27} i \pi .
\end{aligned}
$$

In eq. (6.5), we have defined $L_{0}=\ln (x)$ and $L_{1}=\ln (1-x)$. We note that these are the only transcendental functions that are needed to describe our result.

Although the results for the remaining helicity configurations are still rather compact, they are much larger than for the $\vec{\lambda}=(++++)$ case. We provide them in electronicallyreadable format in the supplementary material. In figure 2 we plot our result for the one-, two- and three-loop finite remainders $\mathcal{M}_{\vec{\lambda}}^{(L)} \equiv\left(\frac{\alpha_{s}}{2 \pi}\right)^{L} f_{\vec{\lambda}}^{(L, \text { fin) }}$ as functions of $x$. We fix $\alpha_{s}=0.118$ and show graphs for the helicity configurations $\vec{\lambda}=(++++),(-+++)$, $(++-+),(--++)$ and $(-+-+)$. All the other helicity amplitudes can be obtained from these through Bose symmetry $(x \leftrightarrow 1-x)$ and parity.

\section{Conclusions}

In this paper, we have computed the helicity amplitudes for the process $g g \rightarrow \gamma \gamma$ in threeloop massless QCD. This is the last missing ingredient required for the calculation of the NNLO QCD corrections to diphoton production in the $g g$ channel. For our analytical threeloop calculation, we have adopted a new projector-based prescription to compute helicity amplitudes in the 't Hooft-Veltman scheme. The expressions at the intermediate stages of our calculation were quite sizable, and we employed recent ideas for the demanding integration-by-parts reductions. Our final results though are remarkably compact. They can be expressed either in terms of standard Harmonic Polylogarithms of weight up to six, or in terms of only 23 transcendental functions defined by up to three-fold sums. This makes the numerical evaluation of our result both fast and numerically stable. Analytical results for both choices of the transcendental functions are provided in the supplementary material that accompany this publication. 

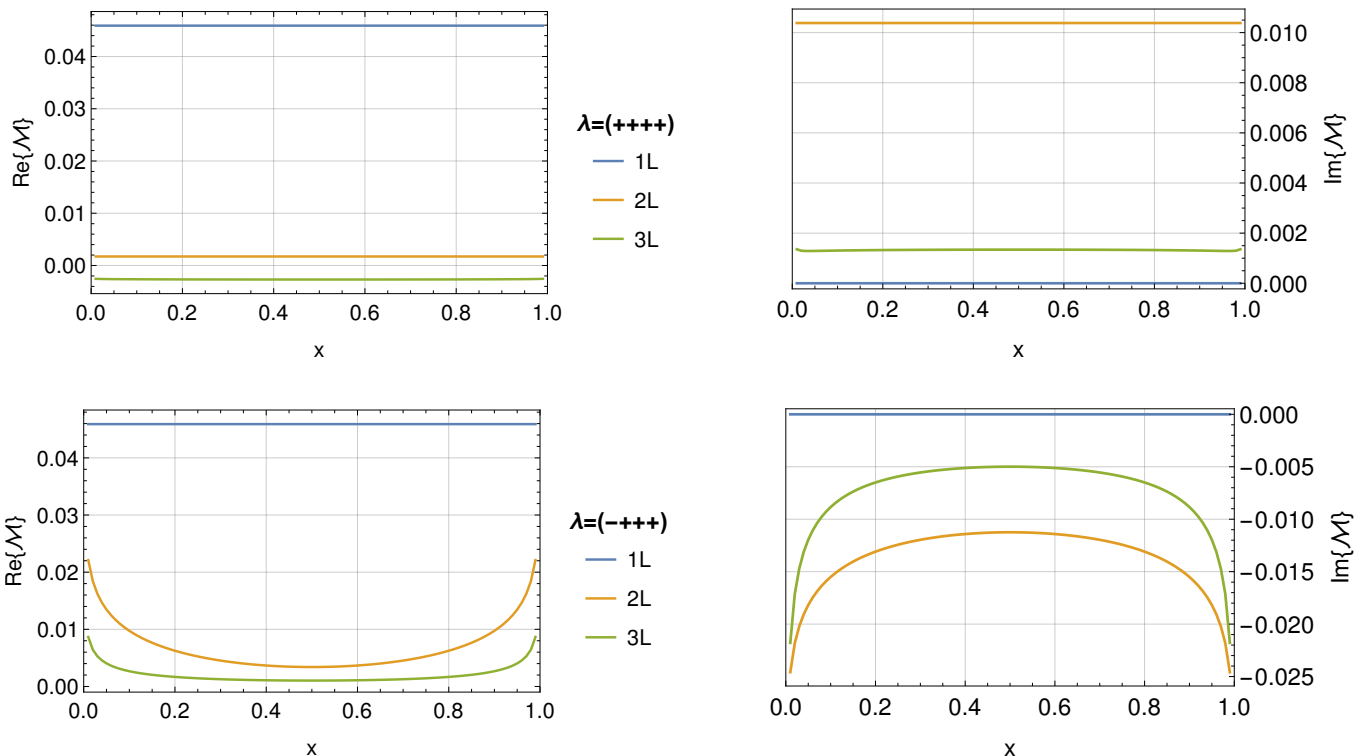

$\lambda=(++++)$

$-1 \mathrm{~L}$

$-2 \mathrm{~L}$

$-3 \mathrm{~L}$

$\lambda=(-++++)$

$-1 \mathrm{~L}$

$-2 \mathrm{~L}$

$-3 \mathrm{~L}$

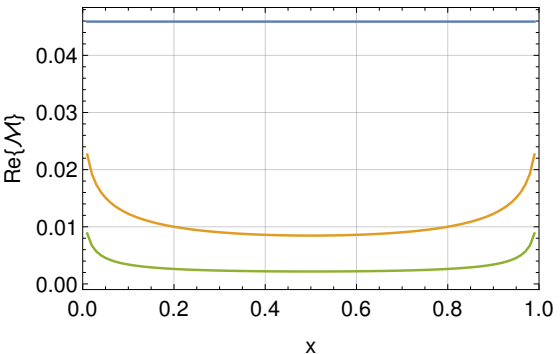

$\lambda=(++-+)$

$-1 \mathrm{~L}$

$-2 \mathrm{~L}$

$-3 \mathrm{~L}$

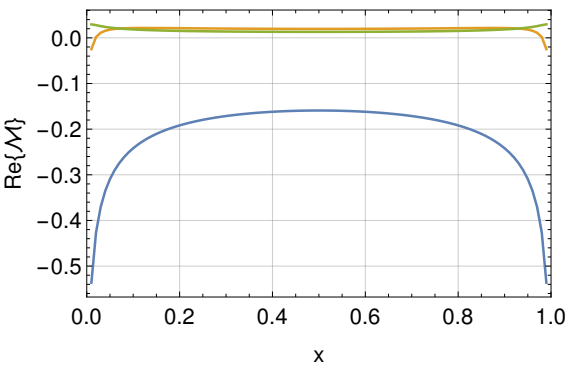

$\lambda=(--++)$

$-1 \mathrm{~L}$

- $2 \mathrm{~L}$

- $3 \mathrm{~L}$
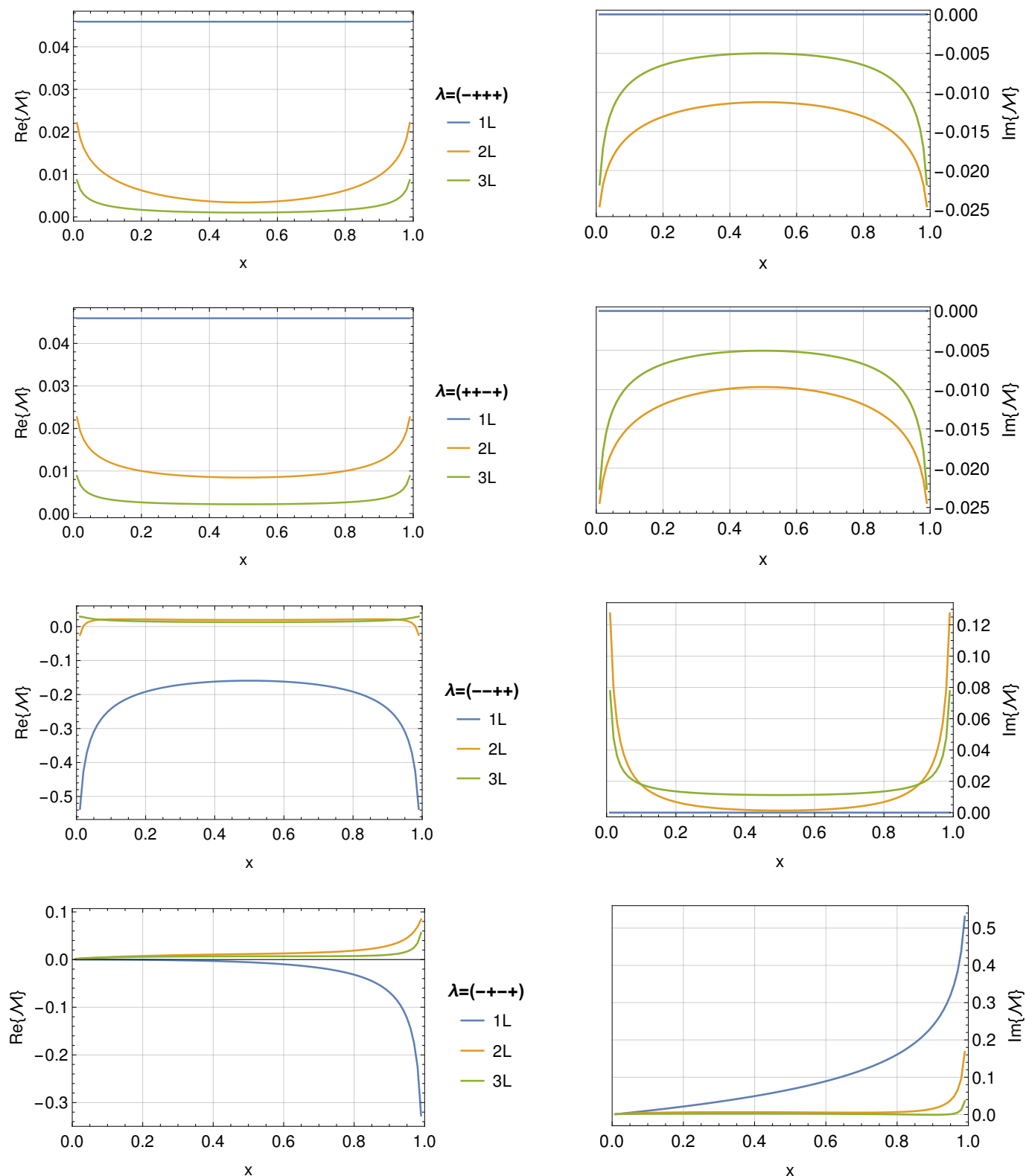

Figure 2. Finite remainders $\mathcal{M}_{\vec{\lambda}}^{(L)} \equiv\left(\frac{\alpha_{s}}{2 \pi}\right)^{L} f_{\vec{\lambda}}^{(L, \text { fin })}$ as functions of $x=-t / s$. 
We envision several possible future directions of investigations. On a more phenomenological side, it would be interesting to combine our results with those of refs $[10,11]$ to obtain NNLO predictions for the $g g \rightarrow \gamma \gamma$ process. On a more theoretical side, the simplicity of our final results begs for an exploration of new ways to perform multiloop calculations. Finally, it would be very interesting to promote our calculation to the fully non-abelian case and consider three-loop scattering amplitudes for the $g g \rightarrow g g$ process. We look forward to pursuing these lines of investigation in the future.

\section{Acknowledgments}

The research of PB and FC was supported by the ERC Starting Grant 804394 HIPQCD and by the U.K. Science and Technology Facilities Council (STFC) under grant ST/T000864/1. AvM was supported in part by the National Science Foundation through Grant 2013859. LT was supported by the Excellence Cluster ORIGINS funded by the Deutsche Forschungsgemeinschaft (DFG, German Research Foundation) under Germany's Excellence Strategy EXC-2094 - 390783311, by the ERC Starting Grant 949279 HighPHun and by the Royal Society grant URF/R1/191125. Feynman graphs were drawn with Jaxodraw [63, 64].

Open Access. This article is distributed under the terms of the Creative Commons Attribution License (CC-BY 4.0), which permits any use, distribution and reproduction in any medium, provided the original author(s) and source are credited.

\section{References}

[1] S. Frixione, Isolated photons in perturbative QCD, Phys. Lett. B 429 (1998) 369 [hep-ph/9801442] [INSPIRE].

[2] S. Catani, L. Cieri, D. de Florian, G. Ferrera and M. Grazzini, Diphoton production at hadron colliders: a fully-differential QCD calculation at NNLO, Phys. Rev. Lett. 108 (2012) 072001 [Erratum ibid. 117 (2016) 089901] [arXiv: 1110.2375] [INSPIRE].

[3] F. Caola, A. Chakraborty, G. Gambuti, A. von Manteuffel and L. Tancredi, Three-loop helicity amplitudes for four-quark scattering in massless QCD, JHEP 10 (2021) 206 [arXiv: 2108.00055] [INSPIRE].

[4] H.A. Chawdhry, M.L. Czakon, A. Mitov and R. Poncelet, NNLO QCD corrections to three-photon production at the LHC, JHEP 02 (2020) 057 [arXiv: 1911.00479] [INSPIRE].

[5] H.A. Chawdhry, M. Czakon, A. Mitov and R. Poncelet, Two-loop leading-color helicity amplitudes for three-photon production at the LHC, JHEP 06 (2021) 150 [arXiv: 2012.13553] [INSPIRE].

[6] H.A. Chawdhry, M. Czakon, A. Mitov and R. Poncelet, Two-loop leading-colour QCD helicity amplitudes for two-photon plus jet production at the LHC, JHEP 07 (2021) 164 [arXiv:2103.04319] [INSPIRE].

[7] H.A. Chawdhry, M. Czakon, A. Mitov and R. Poncelet, NNLO QCD corrections to diphoton production with an additional jet at the LHC, JHEP 09 (2021) 093 [arXiv:2105. 06940] [INSPIRE]. 
[8] B. Agarwal, F. Buccioni, A. von Manteuffel and L. Tancredi, Two-loop leading colour QCD corrections to $q \bar{q} \rightarrow \gamma \gamma g$ and $q g \rightarrow \gamma \gamma q$, JHEP 04 (2021) 201 [arXiv:2102.01820] [INSPIRE].

[9] B. Agarwal, F. Buccioni, A. von Manteuffel and L. Tancredi, Two-loop helicity amplitudes for diphoton plus jet production in full color, Phys. Rev. Lett. 127 (2021) 262001 [arXiv: 2105. 04585] [INSPIRE].

[10] S. Badger et al., Virtual QCD corrections to gluon-initiated diphoton plus jet production at hadron colliders, JHEP 11 (2021) 083 [arXiv:2106.08664] [INSPIRE].

[11] S. Badger, T. Gehrmann, M. Marcoli and R. Moodie, Next-to-leading order QCD corrections to diphoton-plus-jet production through gluon fusion at the LHC, Phys. Lett. B $\mathbf{8 2 4}$ (2022) 136802 [arXiv:2109.12003] [INSPIRE].

[12] C. Anastasiou, E.W.N. Glover and M.E. Tejeda-Yeomans, Two loop QED and QCD corrections to massless fermion boson scattering, Nucl. Phys. B 629 (2002) 255 [hep-ph/0201274] [INSPIRE].

[13] J.M. Campbell, R.K. Ellis, Y. Li and C. Williams, Predictions for diphoton production at the LHC through NNLO in QCD, JHEP 07 (2016) 148 [arXiv: 1603.02663] [INSPIRE].

[14] T. Gehrmann, N. Glover, A. Huss and J. Whitehead, Scale and isolation sensitivity of diphoton distributions at the LHC, JHEP 01 (2021) 108 [arXiv: 2009.11310] [INSPIRE].

[15] M. Grazzini, S. Kallweit and M. Wiesemann, Fully differential NNLO computations with MATRIX, Eur. Phys. J. C 78 (2018) 537 [arXiv:1711.06631] [INSPIRE].

[16] S. Alioli et al., Precise predictions for photon pair production matched to parton showers in GENEVA, JHEP 04 (2021) 041 [arXiv:2010.10498] [INSPIRE].

[17] T. Neumann, The diphoton $q_{T}$ spectrum at $N^{3} L L^{\prime}+N N L O$, Eur. Phys. J. C 81 (2021) 905 [arXiv:2107.12478] [INSPIRE].

[18] Z. Bern, A. De Freitas and L.J. Dixon, Two loop amplitudes for gluon fusion into two photons, JHEP 09 (2001) 037 [hep-ph/0109078] [INSPIRE].

[19] Z. Bern, L.J. Dixon and C. Schmidt, Isolating a light Higgs boson from the diphoton background at the CERN LHC, Phys. Rev. D 66 (2002) 074018 [hep-ph/0206194] [INSPIRE].

[20] CMS collaboration, Measurement of differential cross sections for the production of a pair of isolated photons in pp collisions at $\sqrt{s}=7 \mathrm{TeV}$, Eur. Phys. J. C 74 (2014) 3129 [arXiv: 1405.7225] [INSPIRE].

[21] ATLAS collaboration, Measurement of the production cross section of pairs of isolated photons in pp collisions at $13 \mathrm{TeV}$ with the ATLAS detector, JHEP 11 (2021) 169 [arXiv:2107.09330] [INSPIRE].

[22] S.P. Martin, Shift in the LHC Higgs diphoton mass peak from interference with background, Phys. Rev. D 86 (2012) 073016 [arXiv:1208.1533] [INSPIRE].

[23] L.J. Dixon and Y. Li, Bounding the Higgs boson width through interferometry, Phys. Rev. Lett. 111 (2013) 111802 [arXiv: 1305.3854] [INSPIRE].

[24] LHC Higgs Cross Section Working Group collaboration, Handbook of LHC Higgs cross sections: 4. Deciphering the nature of the Higgs sector, arXiv:1610.07922 [INSPIRE].

[25] S.P. Martin, Interference of Higgs diphoton signal and background in production with a jet at the LHC, Phys. Rev. D 88 (2013) 013004 [arXiv:1303.3342] [InSPIRE]. 
[26] D. de Florian, N. Fidanza, R.J. Hernández-Pinto, J. Mazzitelli, Y. Rotstein Habarnau and G.F.R. Sborlini, A complete $O\left(\alpha_{S}^{2}\right)$ calculation of the signal-background interference for the Higgs diphoton decay channel, Eur. Phys. J. C 73 (2013) 2387 [arXiv:1303.1397] [INSPIRE].

[27] F. Coradeschi et al., Interference effects in the $H(\rightarrow \gamma \gamma)+2$ jets channel at the LHC, Phys. Rev. D 92 (2015) 013004 [arXiv: 1504.05215] [InSPIRE].

[28] J. Campbell, M. Carena, R. Harnik and Z. Liu, Interference in the $g g \rightarrow h \rightarrow \gamma \gamma$ on-shell rate and the Higgs boson total width, Phys. Rev. Lett. 119 (2017) 181801 [Addendum ibid. 119 (2017) 199901] [arXiv: 1704.08259] [INSPIRE].

[29] T. Peraro and L. Tancredi, Physical projectors for multi-leg helicity amplitudes, JHEP 07 (2019) 114 [arXiv: 1906.03298] [INSPIRE].

[30] T. Peraro and L. Tancredi, Tensor decomposition for bosonic and fermionic scattering amplitudes, Phys. Rev. D 103 (2021) 054042 [arXiv: 2012.00820] [InSPIRE].

[31] G. 't Hooft and M.J.G. Veltman, Regularization and renormalization of gauge fields, Nucl. Phys. B 44 (1972) 189 [INSPIRE].

[32] T. Binoth, E.W.N. Glover, P. Marquard and J.J. van der Bij, Two loop corrections to light by light scattering in supersymmetric QED, JHEP 05 (2002) 060 [hep-ph/0202266] [INSPIRE].

[33] L.J. Dixon, Calculating scattering amplitudes efficiently, in Theoretical Advanced Study Institute in Elementary Particle Physics (TASI 95): QCD and beyond, (1996), pg. 539 [hep-ph/9601359] [INSPIRE].

[34] J. Henn, B. Mistlberger, V.A. Smirnov and P. Wasser, Constructing d-log integrands and computing master integrals for three-loop four-particle scattering, JHEP 04 (2020) 167 [arXiv: 2002.09492] [INSPIRE].

[35] F. Caola, A. Von Manteuffel and L. Tancredi, Diphoton amplitudes in three-loop quantum chromodynamics, Phys. Rev. Lett. 126 (2021) 112004 [arXiv:2011.13946] [INSPIRE].

[36] K.G. Chetyrkin and F.V. Tkachov, Integration by parts: the algorithm to calculate $\beta$-functions in 4 loops, Nucl. Phys. B 192 (1981) 159 [INSPIRE].

[37] S. Laporta, High precision calculation of multiloop Feynman integrals by difference equations, Int. J. Mod. Phys. A 15 (2000) 5087 [hep-ph/0102033] [INSPIRE].

[38] P. Nogueira, Automatic Feynman graph generation, J. Comput. Phys. 105 (1993) 279 [INSPIRE].

[39] A. von Manteuffel and C. Studerus, Reduze 2 - distributed Feynman integral reduction, arXiv: 1201.4330 [INSPIRE].

[40] C. Studerus, Reduze-Feynman integral reduction in $C++$, Comput. Phys. Commun. 181 (2010) 1293 [arXiv: 0912.2546] [INSPIRE].

[41] J.A.M. Vermaseren, New features of FORM, math-ph/0010025 [INSPIRE].

[42] A. von Manteuffel and R.M. Schabinger, Quark and gluon form factors to four-loop order in QCD: the $N_{f}^{3}$ contributions, Phys. Rev. D 95 (2017) 034030 [arXiv:1611.00795] [INSPIRE].

[43] J. Gluza, K. Kajda and D.A. Kosower, Towards a basis for planar two-loop integrals, Phys. Rev. D 83 (2011) 045012 [arXiv: 1009.0472] [INSPIRE].

[44] H. Ita, Two-loop integrand decomposition into master integrals and surface terms, Phys. Rev. $D 94$ (2016) 116015 [arXiv: 1510.05626] [INSPIRE]. 
[45] K.J. Larsen and Y. Zhang, Integration-by-parts reductions from unitarity cuts and algebraic geometry, Phys. Rev. D 93 (2016) 041701 [arXiv:1511.01071] [INSPIRE].

[46] J. Böhm, A. Georgoudis, K.J. Larsen, M. Schulze and Y. Zhang, Complete sets of logarithmic vector fields for integration-by-parts identities of Feynman integrals, Phys. Rev. D 98 (2018) 025023 [arXiv: 1712.09737] [INSPIRE].

[47] R.M. Schabinger, A new algorithm for the generation of unitarity-compatible integration by parts relations, JHEP 01 (2012) 077 [arXiv:1111.4220] [INSPIRE].

[48] B. Agarwal, S.P. Jones and A. von Manteuffel, Two-loop helicity amplitudes for $g g \rightarrow Z Z$ with full top-quark mass effects, JHEP 05 (2021) 256 [arXiv:2011.15113] [INSPIRE].

[49] A. von Manteuffel and R.M. Schabinger, A novel approach to integration by parts reduction, Phys. Lett. B $\mathbf{7 4 4}$ (2015) 101 [arXiv: 1406 .4513] [INSPIRE].

[50] T. Peraro, Scattering amplitudes over finite fields and multivariate functional reconstruction, JHEP 12 (2016) 030 [arXiv:1608.01902] [INSPIRE].

[51] T. Peraro, FiniteFlow: multivariate functional reconstruction using finite fields and dataflow graphs, JHEP 07 (2019) 031 [arXiv: 1905.08019] [INSPIRE].

[52] J. Vollinga and S. Weinzierl, Numerical evaluation of multiple polylogarithms, Comput. Phys. Commun. 167 (2005) 177 [hep-ph/0410259] [INSPIRE].

[53] T. Gehrmann and E. Remiddi, Numerical evaluation of harmonic polylogarithms, Comput. Phys. Commun. 141 (2001) 296 [hep-ph/0107173] [INSPIRE].

[54] C. Duhr and F. Dulat, PolyLogTools - polylogs for the masses, JHEP 08 (2019) 135 [arXiv: 1904.07279] [INSPIRE].

[55] J.M. Henn, Multiloop integrals in dimensional regularization made simple, Phys. Rev. Lett. 110 (2013) 251601 [arXiv: 1304.1806] [INSPIRE].

[56] S. Catani, The singular behavior of QCD amplitudes at two loop order, Phys. Lett. B 427 (1998) 161 [hep-ph/9802439] [INSPIRE].

[57] R.V. Harlander, Virtual corrections to $g g \rightarrow H$ to two loops in the heavy top limit, Phys. Lett. B 492 (2000) 74 [hep-ph/0007289] [InSPIRE].

[58] F. Cascioli, P. Maierhofer and S. Pozzorini, Scattering amplitudes with open loops, Phys. Rev. Lett. 108 (2012) 111601 [arXiv: 1111.5206] [INSPIRE].

[59] F. Buccioni et al., OpenLoops 2, Eur. Phys. J. C 79 (2019) 866 [arXiv:1907.13071] [INSPIRE].

[60] J.M. Campbell, R.K. Ellis and C. Williams, Vector boson pair production at the LHC, JHEP 07 (2011) 018 [arXiv: 1105.0020] [inSPIRE].

[61] R. Boughezal et al., Color singlet production at NNLO in MCFM, Eur. Phys. J. C 77 (2017) 7 [arXiv: 1605.08011] [INSPIRE].

[62] C. Duhr, H. Gangl and J.R. Rhodes, From polygons and symbols to polylogarithmic functions, JHEP 10 (2012) 075 [arXiv:1110.0458] [inSPIRE].

[63] D. Binosi and L. Theussl, JaxoDraw: a graphical user interface for drawing Feynman diagrams, Comput. Phys. Commun. 161 (2004) 76 [hep-ph/0309015] [INSPIRE].

[64] J.A.M. Vermaseren, Axodraw, Comput. Phys. Commun. 83 (1994) 45 [INSPIRE]. 